\title{
Sample Collection into Sterile Vacuum Tubes to Preserve Arsenic Speciation in Natural Water Samples
}

\author{
Orhan Gunduz ${ }^{1}$; Hakan Gurleyuk ${ }^{2}$; Ayse Cakir ${ }^{3}$; Alper Elci ${ }^{4}$; Alper Baba ${ }^{5}$; and Celalettin Simsek ${ }^{6}$
}

\begin{abstract}
The accurate speciation analysis of arsenic is a serious concern for water quality monitoring programs. Because the preservation of sample integrity until analysis is one of the most important aspects of speciation, this study aims to compare the performance of four different sample preservation methods under diverse conditions of sample quality. Natural samples with different characteristics were collected into the following containers to study their effectiveness: (1) standard high-density polyethylene bottle with no preservative; (2) empty, sterile Vacuette tube with no preservative; (3) sterile tripotassium ethylenediaminetetraacetic acid (K3EDTA) Vacuette tube with K3EDTA additive; and (4) empty, sterile Vacuette tube with added hydrochloric acid ( $\mathrm{HCl})$. Known concentrations of arsenite [As(III)] were also added to each container to monitor the oxidation of As(III) to arsenate [As(V)]. The results revealed recovery ratios exceeding 95\% in all containers with sterile vacuum conditions. In particular, the K3EDTA Vacuette tube yielded a recovery very close to $100 \%$ of the spiked As(III), which is known to rapidly oxidize to As(V). Overall, collecting the sample into a container under sterile vacuum conditions and using a universally accepted preserving agent such as EDTA or $\mathrm{HCl}$ significantly improved the preservation of the original species distribution in the water matrix studied, compared to sampling without the use of preservation methods. After validation by future research, these sterile vacuum tubes can possibly be utilized for collecting and storing samples for the routine speciation analysis of other elements such as selenium, chromium, and antimony. DOI: 10.1061/(ASCE)EE.1943-7870.0000717. () 2013 American Society of Civil Engineers.
\end{abstract}

CE Database subject headings: Groundwater management; Arsenic; Sampling; Water quality.

Author keywords: Groundwater; Arsenic; Speciation; Field preservation; Sterile vacuum tubes.

\section{Introduction}

Arsenic occurs in natural waters in inorganic [i.e., arsenite, As(III), and arsenate, $\mathrm{As}(\mathrm{V})]$ and organic [i.e., monomethyl arsonate (MMA) and dimethyl arsenate (DMA)] forms (Cullen and Reimer 1989; Smedley and Kinniburgh 2002; Bhattacharya et al. 2007). When compared to their inorganic counterparts, the organic forms are typically observed at much lower concentrations (Kim et al. 2007). Inorganic $\operatorname{As}(\mathrm{V})$ is the major arsenic species in many groundwater and surface water resources where oxidizing conditions prevail, whereas inorganic As(III) is typically found to occur in groundwater that is mostly under reducing conditions (Le et al. 2000; Markley and Herbert 2009). Redox potential and $\mathrm{pH}$ are the most important environmental factors controlling arsenic speciation (Terlecka 2005). Depending on these factors, the ionic charges of inorganic arsenic species vary from 0 to- 3 (Kim et al. 2007). The

${ }^{1}$ Dept. of Environmental Engineering, Dokuz Eylul Univ., Izmir 35160, Turkey (corresponding author). E-mail: orhan.gunduz@deu.edu.tr

${ }^{2}$ Applied Speciation and Consulting, LLC, Bothell, WA 98011.

${ }^{3}$ Graduate School of Natural and Applied Sciences, Dokuz Eylul Univ., Izmir 35160, Turkey.

${ }^{4}$ Dept. of Environmental Engineering, Dokuz Eylul Univ., Izmir 35160, Turkey.

${ }^{5}$ Dept. of Civil Engineering, Izmir Institute of Technology, Izmir 35430, Turkey.

${ }^{6}$ Dept. of Drilling, Torbali Vocational School, Dokuz Eylul Univ., Izmir 35850, Turkey.

Note. This manuscript was submitted on March 27, 2012; approved on April 10, 2013; published online on April 12, 2013. Discussion period open until January 1, 2014; separate discussions must be submitted for individual papers. This paper is part of the Journal of Environmental Engineering, Vol. 139, No. 8, August 1, 2013. (C) ASCE, ISSN 0733-9372/2013/8-1080$1088 / \$ 25.00$. mobility and toxicity of arsenic are also closely related to its oxidation states (Hung et al. 2004). The toxicity of different arsenic species varies; As(III) has the highest toxicity among other forms [Jain and Ali 2000; Bissen and Frimmel 2003; Agency for Toxic Substances and Disease Registry (ATSDR) 2007] because of its ability to bind with sulfhydryl groups (Markley and Herbert 2009). The organic species, on the other hand, are considered to be the least toxic (Miller et al. 2000). The strong link between the form of arsenic and its toxicity level makes speciation an important concern for domestic water supplies.

A vast number of methods exist for the analysis of arsenic species (Jain and Ali 2000; Smedley and Kinniburgh 2002; Gong et al. 2002). These methods have been developed to distinguish among different forms under field and laboratory conditions (Kumaresan and Riyazuddin 2001; Garbarino et al. 2002). Field speciation analysis techniques are mostly based on the principle of isolating and separating a known species on some medium via solid-phase extraction methods (Yalcin and Le 2001). The species trapped in the medium and passed to the eluent are separately analyzed by standard methods used in total arsenic determination, as discussed by Melamed (2005). Laboratory speciation techniques, however, do not require that the species are separated from each other in field conditions and are based on the principle of measuring each species with one of the hyphenated methods such as ion chromatographyinductively coupled plasma mass spectrometry (IC-ICP-MS) and ion chromatography-hydride generation atomic fluorescence spectrometry (IC-HG-AFS) (Kumaresan and Riyazuddin 2001; Garbarino et al. 2002; Terlecka 2005), which can provide very low detection limits (Kim et al. 2007).

Analytical laboratory speciation methods have the advantage of accurately determining $\mathrm{As}(\mathrm{III}), \mathrm{As}(\mathrm{V}), \mathrm{MMA}$, and DMA by using a sample volume of less than $200 \mu \mathrm{L}$ without any prior sample preparation; these require high-tech hyphenated instruments and 
skilled personnel. However, if the original species distribution in the sample cannot be preserved until analysis, these methods and instrumentation are all useless. On the other hand, field speciation methods typically separate only inorganic species with solid-phase extraction cartridges, in which species are separated to remain in the solid matrix or pass to the eluent based on their ionic charge, after which they are independently analyzed by classical total arsenic analysis. The disadvantage to field methods is primarily that the composition of the sample matrix can be higher than cartridge capacity, which would necessitate a larger capacity cartridge. Moreover, the requirement to precondition the exchange cartridge and to extract the retained arsenic from the cartridge increases the laboratory work to be done. Finally, the necessity to make two separate analytical determinations for As(III) and As (V) makes field speciation methods rather inconvenient and impractical for assessment and characterization missions. Because of these drawbacks, it is typically accepted that the best method to speciate arsenic is to preserve, store, and transport the original sample with minimum external interference and to use laboratory speciation methods.

In most natural waters, inorganic arsenic species dominate in the liquid matrix; thus, the speciation analysis of arsenic is generally aimed at determining the relative concentrations of As(III) and As $(\mathrm{V})$ in water. This is also consistent with the requirements of public health specialists, who require the relative levels of the two most toxic arsenic species for risk assessment and exposure analysis (EPA 1999; Smith et al. 2000; Abernathy et al. 2003; Akter et al. 2005; Kapaj et al. 2006; Tapio and Grosche 2006). The major problem with the inorganic arsenic forms is the rapid oxidation of As (III) to $\mathrm{As}(\mathrm{V})$ in the presence of oxygen (Kumar and Riyazuddin 2010). Consequently, the preservation and stabilization of inorganic arsenic species has been a major problem in arsenic speciation analysis, particularly in natural waters, until Bednar et al. (2002) proposed the use of opaque bottles with preservation by ethylenediaminetetraacetic acid (EDTA). It has been reported that the distribution of arsenic species changes within two days (with or without exposure to sunlight) when proper preservation and stabilization measures are not taken (Bednar et al. 2002). Thus, sample preservation is of utmost importance for the accurate and reliable quantification of arsenic species in water samples.

The most common approach to preserve As species in solution includes the addition of acidic solutions of hydrochloric acid $(\mathrm{HCl})$, nitric acid $\left(\mathrm{HNO}_{3}\right)$, sulfuric acid $\left(\mathrm{H}_{2} \mathrm{SO}_{4}\right)$, phosphoric acid $\left(\mathrm{H}_{3} \mathrm{PO}_{4}\right)$, nitriloacetic acid, and ascorbic acid in addition to complexing agents such as EDTA in combination with temperature control (Oliveira et al. 2006; Samanta and Clifford 2006). For example, Gallagher et al. (2001) reported that it was necessary to lower the sample $\mathrm{pH}$ to approximately 3.2 with acetic acid to preserve the arsenic species when using EDTA in samples containing iron (III) $[\mathrm{Fe}(\mathrm{III})]$. However, according to Bednar et al. (2002), disodium EDTA may preserve the arsenic species in the presence of $\mathrm{Fe}(\mathrm{III})$-containing groundwater without lowering the $\mathrm{pH}$ of samples in opaque bottles. Among these alternative methods, preservation with $\mathrm{HCl}$ and EDTA have found a wider range of application; these are now considered to be the two most commonly applied preservation agents in arsenic speciation analysis (Garbarino et al. 2002).

Regardless of the preserving agent added, the exposure of samples to air (i.e., oxygen) creates conditions in which inorganic As (III) can quickly oxidize into As(V). Particularly in samples with very low redox potentials, even small amounts of oxygen can alter the redox conditions in the sample and change the form of the arsenic species. Similarly, maintaining a cold storage chain for minimizing microbial activity in the sample is another important aspect of preserving the distribution of original species in the sample (Garbarino et al. 2002). Finally, prevention of exposure to sunlight is also a fundamental aspect of sample preservation because sunlight may stimulate certain photochemical reactions that can alter the geochemistry of the water sample.

Vacuum sample tubes are the most common sample containers used in collecting and preserving human blood samples for biochemical analysis (Grenier Bio-One 2007). These tubes are evacuated to create a vacuum inside the tube, facilitating the draw of liquid, and may contain different additives designed to stabilize and preserve the specimen prior to analytical testing. The Vacuette tubes used in this study are also under vacuum and have no contact with atmospheric oxygen, which is one of factors that may affect the speciation of arsenic. These tubes are cheaper than the common high-density polyethylene (HDPE) or low-density polyethylene (LDPE) environmental sample containers. Because Vacuette tubes can hold only up to $10 \mathrm{~mL}$ of solution, they are more applicable when no more than $5 \mathrm{~mL}$ of sample is required for analysis. As a result of their limited volume, shipping costs can be significantly reduced because all speciation samples require overnight shipping to the laboratory after collection and thousands of these tubes can be stored in a single refrigerator shelf.

Based on this premise, the goal of this study is to evaluate the applicability of these tubes for sample collection, storage, and transportation in monitoring arsenic speciation in surface and subsurface waters. To achieve this objective, natural water samples and natural water samples spiked with a known concentration of As(III) were collected into different types of sample containers. The speciation of arsenic in these samples was determined in the laboratory with IC-ICP-MS. The recovery of the spiked As(III) and the interconversions and losses of the species in each sample were used as the benchmarks for the suitability of each sample container.

\section{Materials and Methods}

\section{Sampling Media and Preserving Agents}

Four different sample containers with distinct characteristics were used in this study to test and compare their effectiveness in preserving arsenic species under ideal and natural conditions. The fundamental data regarding the containers used in field sampling and preservation are given in Table 1. Thus, regular 50-mL HDPE bottles used in routine environmental sampling programs were compared with three different types of vacuum (Vacuette) sample tubes (Grenier Bio-One 2007), resulting in a comparison of four containers: (1) HDPE bottle with no additive; (2) empty Vacuette tube (white cap) with no additive; (3) tripotassium (K3) EDTA Vacuette tube (purple cap) with built-in K3EDTA additive; and (4) $\mathrm{HCl}$ added to an empty Vacuette tube (white cap). Among these containers, all except the HDPE bottle is sealed under vacuum and contains

Table 1. Containers Used in Field Sampling and Preservation

\begin{tabular}{lccc}
\hline Tube/bottle & Status & Material & $\begin{array}{c}\text { Preserving } \\
\text { agent }\end{array}$ \\
\hline $\begin{array}{l}\text { HDPE bottle } \\
(50 \mathrm{~mL})\end{array}$ & $\begin{array}{c}\text { Atmospheric } \\
\text { pressure }\end{array}$ & HDPE & None \\
$\begin{array}{l}\text { Vacuette tube } \\
(10 \mathrm{~mL})\end{array}$ & Vacuum & $\begin{array}{l}\text { Polyethylene } \\
\text { terephthalate }\end{array}$ & None \\
$\begin{array}{l}\text { Vacuette tube } \\
(10 \mathrm{~mL})\end{array}$ & Vacuum & $\begin{array}{l}\text { Polyethylene } \\
\text { terephthalate } \\
\text { Vacuette tube }\end{array}$ & K3EDTA \\
$(10 \mathrm{~mL})$ & Vacuum & $\begin{array}{l}\text { Polyethylene } \\
\text { terephthalate }\end{array}$ & $\mathrm{HCl}$ \\
\hline
\end{tabular}


no air inside. All liquid matrices [i.e., sample, stock arsenic solution, and preserving agent $(\mathrm{HCl})]$ were added to the Vacuette tubes by using 10-mL polyethylene (PE) sterile syringes, similar to blood collection procedures, as shown in Fig. 1.

\section{Characteristics of Field Samples}

The field application was conducted in the Simav Plain, located in the Kutahya Province of Turkey, during February of 2011. The site was previously reported to have high arsenic levels in the groundwater samples collected from the alluvial plain (Gunduz et al. 2010). Three samples were selected from the Simav Plain to cover a wide range of redox conditions and hydrogeochemical patterns. The physical parameters of these field samples are given in Table 2. Accordingly, three samples used in this study were collected from wells drilled to different depths and represented oxidizing, neutral, and reducing conditions. The field parameters of the samples (temperature, $\mathrm{pH}$, electrical conductivity, oxidation reduction potential, and dissolved oxygen) were measured in situ with a portable multi-parameter probe (Hach-Lange HQ40d).

The major ion compositions of these samples are also given in Table 2. Major anion and cation analyses were performed with IC (Dionex ICS-3000). The first sample was from a shallowly dug well in the unconfined aquifer, which has high ionic strength and was under the influence of geothermal fluid intrusion, as was obvious from the comparably high electrical conductivity value. The second sample was also from the unconfined aquifer, but has moderate ionic strength. On the other hand, the third sample was from an artesian well that was drilled to the confined aquifer, which has moderate ionic strength under reducing conditions.
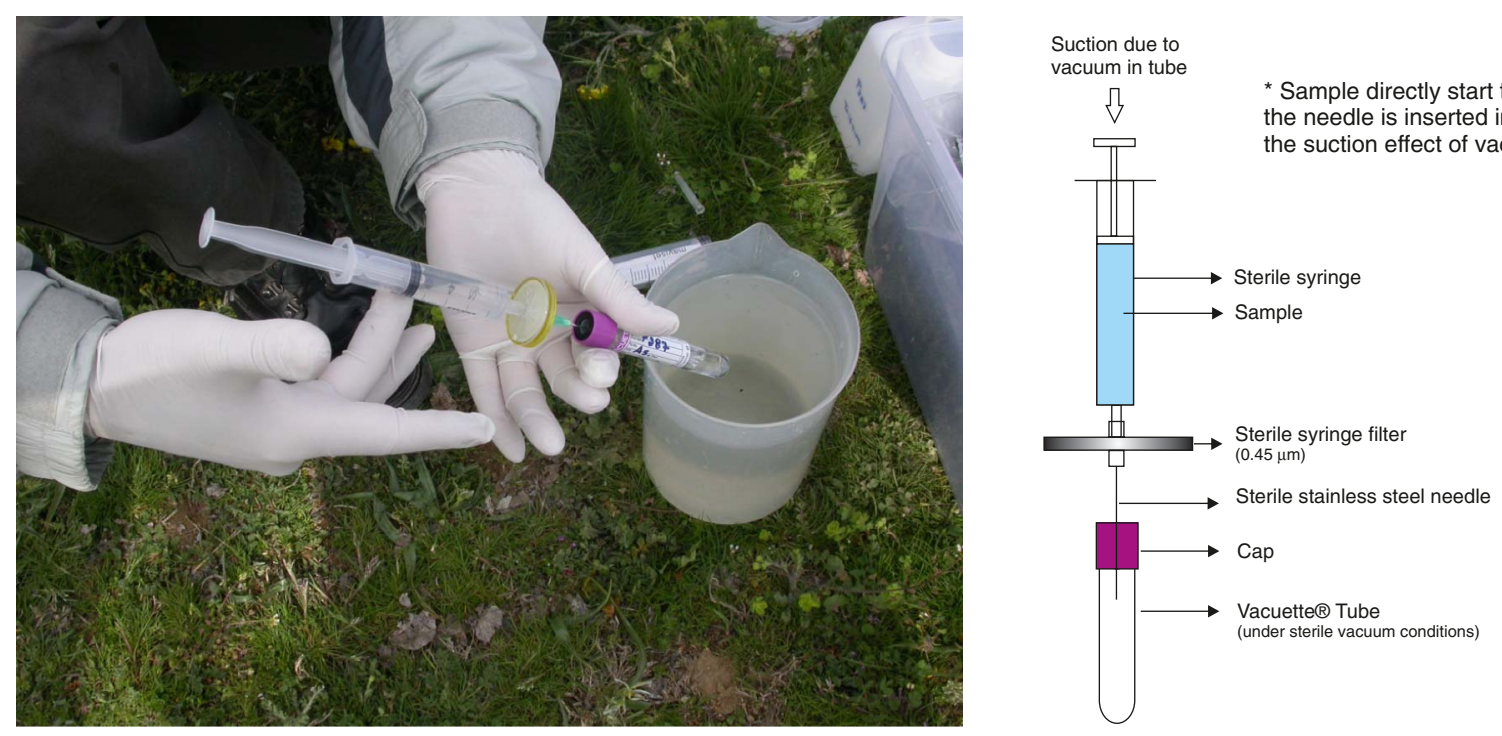

Fig. 1. Sample injection into vacuum tubes

Table 2. Physical Parameters and Major Ion Concentrations of Field Samples

\begin{tabular}{|c|c|c|c|c|}
\hline Parameter $^{\mathrm{a}}$ & & Sample 1: & Sample 2: & Sample 3: \\
\hline Sample type & Unit & Shallow well (unconfined aquifer) & Deep well (unconfined aquifer) & Artesian well (confined aquifer) \\
\hline Temp & ${ }^{\circ} \mathrm{C}$ & 15.3 & 14.8 & 15.3 \\
\hline pH & - & 7.05 & 8.06 & 7.96 \\
\hline ORP & $\mathrm{mV}$ & 68.3 & 3.2 & -80.8 \\
\hline $\mathrm{EC}$ & $\mu \mathrm{S} \mathrm{cm}^{-1}$ & 3050 & 702 & 776 \\
\hline DO & $\mathrm{mg} \mathrm{L}^{-1}$ & 2.14 & 2.45 & 3.94 \\
\hline DO & $\%$ & 23.3 & 26.8 & 44.8 \\
\hline $\mathrm{F}^{-}$ & $\mathrm{mg} \mathrm{L}^{-1}$ & 4.306 & 0.192 & 0.083 \\
\hline $\mathrm{Cl}^{-}$ & $\mathrm{mg} \mathrm{L}^{-1}$ & 72.596 & 6.170 & 3.408 \\
\hline $\mathrm{NO}_{2}^{-}$ & $\mathrm{mg} \mathrm{L}^{-1}$ & $<0.5$ & $<0.5$ & $<0.5$ \\
\hline $\mathrm{Br}^{-}$ & $\mathrm{mg} \mathrm{L}^{-1}$ & 0.396 & 0.196 & 0.176 \\
\hline $\mathrm{NO}_{3}^{-}$ & $\mathrm{mg} \mathrm{L}^{-1}$ & $<0.5$ & $<0.5$ & $<0.5$ \\
\hline $\mathrm{PO}_{4}^{-3}$ & $\mathrm{mg} \mathrm{L}^{-1}$ & $<1.0$ & $<1.0$ & $<1.0$ \\
\hline $\mathrm{SO}_{4}^{-2}$ & $\mathrm{mg} \mathrm{L}^{-1}$ & 880.539 & 53.555 & 0.060 \\
\hline $\mathrm{HCO}_{3}^{-}$ & $\mathrm{mg} \mathrm{L}^{-1}$ & 871.080 & 414.800 & 512.400 \\
\hline $\mathrm{Li}^{+}$ & $\mathrm{mg} \mathrm{L}^{-1}$ & 1.445 & 0.001 & 0.004 \\
\hline $\mathrm{Na}^{+}$ & $\mathrm{mg} \mathrm{L}^{-1}$ & 283.069 & 10.457 & 51.655 \\
\hline $\mathrm{NH}_{4}^{+}$ & $\mathrm{mg} \mathrm{L}^{-1}$ & 0.976 & 1.303 & 12.905 \\
\hline $\mathrm{K}^{+}{ }^{4}$ & $\mathrm{mg} \mathrm{L}^{-1}$ & 37.014 & 1.140 & 1.994 \\
\hline $\mathrm{Mg}^{+2}$ & $\mathrm{mg} \mathrm{L}^{-1}$ & 40.080 & 36.333 & 21.552 \\
\hline $\mathrm{Ca}^{+2}$ & $\mathrm{mg} \mathrm{L}^{-1}$ & 176.336 & 99.342 & 84.583 \\
\hline TOC & $\mathrm{mg} \mathrm{L}^{-1}$ & 20.900 & 4.190 & 9.236 \\
\hline
\end{tabular}

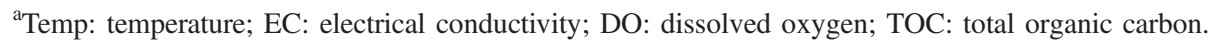


These sampling sites are situated approximately $4 \mathrm{~km}$ apart and the total arsenic concentrations were previously reported to be 77.0, 124.2, and $217.0 \mu \mathrm{g} \mathrm{L}^{-1}$, respectively (Gunduz et al. 2010). Accordingly, arsenite spike concentrations were achieved at 115 and $230 \mu \mathrm{g} \mathrm{L}^{-1}$.

\section{Preparation of Stock Solutions and Preserving Agents}

Lab-grade chemicals were used in preparing the chemical solutions used in this study. All solutions were prepared with deionized (DI) water (Millipore Milli-Q Gradient Water Purification System), which was later deoxygenated with nitrogen $\left(\mathrm{N}_{2}\right)$ gas for 30 min to minimize the risk of $\mathrm{As}(\mathrm{III})$ oxidation to $\mathrm{As}(\mathrm{V})$ as a result of oxygen present in the DI water used in solution preparation. Stock solutions of As(III) were prepared from laboratory reagent grade $(98+\%)$ sodium arsenite (Fisher Scientific product code: $\mathrm{S} / 2320 / 48$ ), packed in powder form. The $\mathrm{HCl}$ used as a preservative agent was prepared from 30\% Suprapur HCL (Merck: 1.00318.1000).

Three stock solutions of As(III) were prepared at concentrations of $1,150,11.5$, and $0.115 \mathrm{mg} \mathrm{L}^{-1}$ by using deoxygenated DI water. The stock solution used for spikes was also analyzed for arsenic speciation and the determined concentration was used to determine recovery values for speciation analysis. A $2 \% \mathrm{HCl}$ solution was also prepared as a preserving agent by using deoxygenated DI water. Once these solutions were prepared, they were stored in glass bottles that were closed with a silicon stopper and cap. The small headspace in the bottle was free from air (and oxygen) because the solution was purged with $\mathrm{N}_{2}$ gas. The extraction of solutions from these bottles under field conditions was done with sterile syringes and needles through the silicon stopper without allowing contact with atmospheric oxygen.

\section{Sample Collection}

In field conditions, samples were first added to a 2-L polypropylene beaker in which field parameters (temperature, $\mathrm{pH}$, electrical conductivity, dissolved oxygen, and oxidation reduction potential) were measured. Meanwhile, samples were filtered and filled into different sampling mediums, as shown in Table 3. In all mediums, the first two trials were reserved for DI water and DI water spiked with a stock solution containing $115 \mu \mathrm{g} \mathrm{L}^{-1}$ of As(III) (i.e., Trials $1,2,9,10,17,18,25$, and 26). The next six trials in each medium were made with three samples (two trials for each sample) collected from the field with and without the addition of stock solutions (i.e., Trials 3-8, 11-16, 19-24, and 27-32). All field samples were filtered through $0.45-\mu \mathrm{m}$ syringe-type filters made from cellulose acetate before injected or filled into their corresponding sampling containers. For Trials 1 through 16, no preserving agent was added. For Trials 17 through 24, the preserving agent K3EDTA was already sprayed into the tubes, and no external addition was made. Thus, only for Trials 25 through $32,2 \% \mathrm{HCl}$ solution was diluted according to the sample volume in different mediums $(10 \mathrm{~mL}$ for Vacuette tubes and $50 \mathrm{~mL}$ for HDPE bottles) to obtain a final concentration of $0.2 \% \mathrm{HCl}$ inside the tube.

All trials with As(III) spikes (i.e., all even-numbered trials) were first prepared in a separate container. A $100 \mathrm{~mL}$ volume of DI water or filtered field sample was added to this container. One milliliter was extracted by a sterile syringe, creating a $99 \mathrm{~mL}$ solution. To this amount, $1 \mathrm{~mL}$ of $11.5 \mathrm{mg} \mathrm{L}^{-1} \mathrm{As}(\mathrm{III})$ stock solution was added by a sterile syringe, which resulted in a final As(III) concentration of $115 \mu \mathrm{g} \mathrm{L}^{-1}$ in the solution. From this solution, $10 \mathrm{~mL}$ was injected into the tubes and $50 \mathrm{~mL}$ was filled into the HDPE bottles. For trials with Sample 3, $2 \mathrm{~mL}$ was extracted by a sterile syringe, creating a $98 \mathrm{~mL}$ solution, to which $2 \mathrm{~mL}$ of $11.5 \mathrm{mg} \mathrm{L}^{-1} \mathrm{As}$ (III) stock solution was added, creating a final As(III) concentration of $230 \mu \mathrm{g} \mathrm{L}^{-1}$ in the solution (i.e., Trials 8, 16, and 24).

In trials with the $\mathrm{HCl}$ additive, a $100 \mathrm{~mL}$ volume of DI water or filtered field sample was added to a separate container. Ten milliliters were extracted by a sterile syringe, creating a $90 \mathrm{~mL}$ solution. To this, $10 \mathrm{~mL}$ of $2 \% \mathrm{HCl}$ solution was added to yield $0.2 \%$ of $\mathrm{HCl}$ solution. From this mixture, $1 \mathrm{~mL}$ was extracted by a sterile syringe, creating a $99 \mathrm{~mL}$ solution. To this amount, $1 \mathrm{~mL}$ of $11.5 \mathrm{mg} \mathrm{L}^{-1} \mathrm{As}$ (III) stock solution was added by a sterile syringe, which resulted in a final As(III) concentration of $115 \mu \mathrm{g} \mathrm{L}^{-1}$ in the solution. Ten milliliters were injected into the tubes and $50 \mathrm{~mL}$ was filled into the HDPE bottles (i.e., Trials 25 through 31). For trials with Sample 3, $2 \mathrm{~mL}$ was extracted by a sterile syringe, creating a $98 \mathrm{~mL}$ solution, to which $2 \mathrm{~mL}$ of $11.5 \mathrm{mg} \mathrm{L}^{-1} \mathrm{As}$ (III) stock solution was added by a sterile syringe, creating a final As(III) concentration of $230 \mu \mathrm{g} \mathrm{L}^{-1}$ in the solution (i.e., Trial 32).

Once a trial was completed, the tube/bottle was placed in a dark, temperature controlled $\left(4^{\circ} \mathrm{C}\right)$ storage container and transferred into the laboratory. All trials were stored in a refrigerator until analysis.

\section{Analysis}

The tubes/bottles from 32 trials were analyzed by IC-ICP-MS for the speciation of inorganic and organic forms of arsenic. Furthermore, all trials were also analyzed for total arsenic and other dissolved metals such as iron and manganese (Mn), which are known to interfere with arsenic compounds in water.

\section{Cross-Contamination from Media, Preserving Agents, and Chemicals}

To avoid the possibility of cross-contamination from the sampling medium, the empty bottles and tubes were analyzed for any residual arsenic originating from the material, and no arsenic was found. Similarly, the $\mathrm{HCl}$ used for preservation was tested and found to contain no arsenic. Finally, the sodium arsenite used in spiking As(III) was also tested and verified that it was lab grade.

\section{Results and Discussion}

In this study, the success of the preservation of arsenic species was primarily assessed based on the relative percentage of the fieldspiked As(III) recovered in the laboratory. The differences in storage containers demonstrate the factors that are important in the preservation, storage, and analysis of arsenic species. In addition to percent recovery, the difference of the sum of species from total dissolved arsenic levels are other measures to evaluate the effectiveness of the methods implemented as a part of this study. Because As (V) was not spiked into the samples, any As(V) recovered in addition to the native $\mathrm{As}(\mathrm{V})$ concentration in the samples should be attributed to oxidation of the spiked As(III).

\section{Other Metals}

Because the results of arsenic speciation are directly influenced by other metals such as aluminum, manganese, and iron, the total dissolved levels of these elements were also analyzed, as shown in Table 3. These results demonstrate their corresponding conditions in DI water and three field samples used in the study. As shown in Table 3, the total arsenic levels in all trials ranged between 30.62 and $41.97 \mu \mathrm{g} \mathrm{L}^{-1}$ for Sample 1; between 92.78 and $105.05 \mu \mathrm{g} \mathrm{L}^{-1}$ for Sample 2; and between 153.45 and $181.17 \mu \mathrm{g} \mathrm{L}^{-1}$ for Sample 3. These levels represented low, moderate, and high 


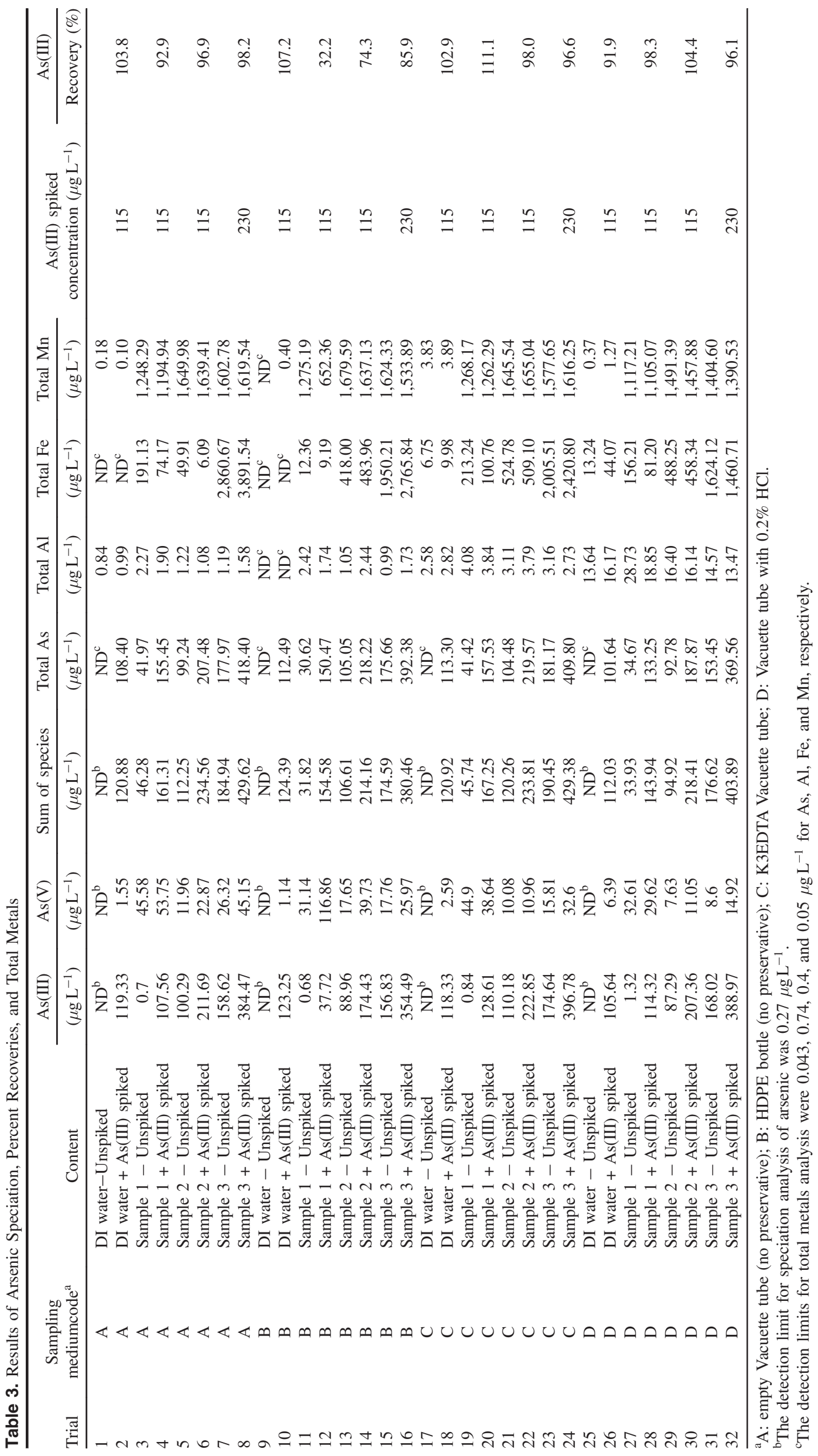

1084 / JOURNAL OF ENVIRONMENTAL ENGINEERING @) ASCE / AUGUST 2013 


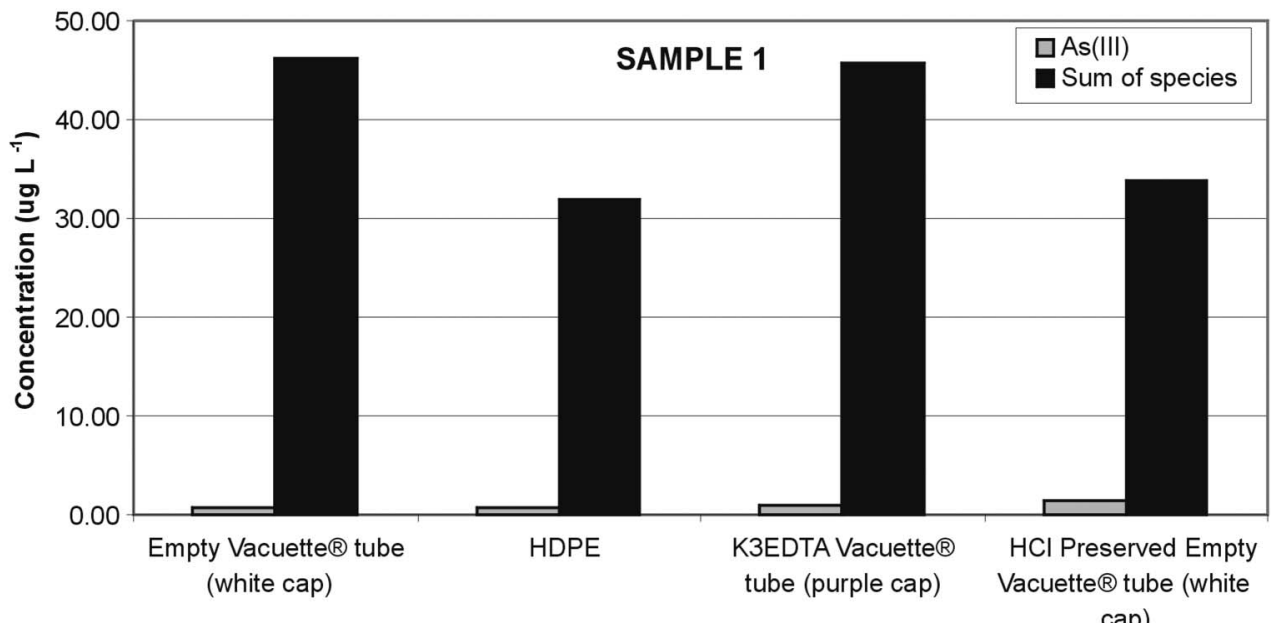

Sampling Media

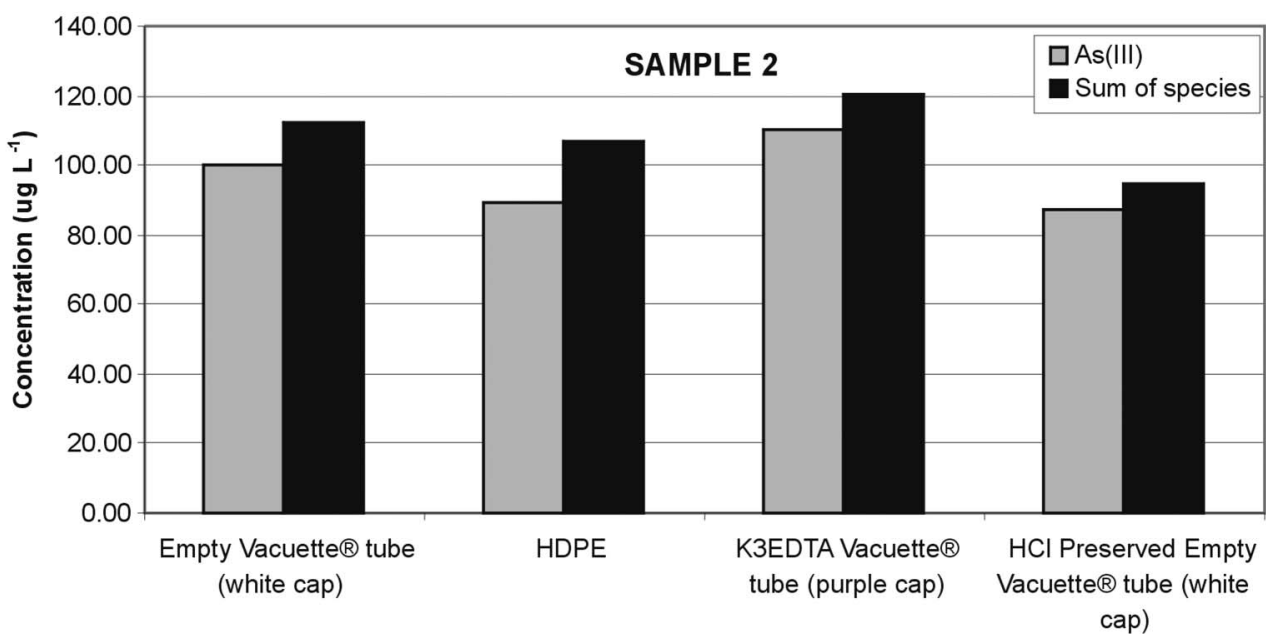

Sampling Media

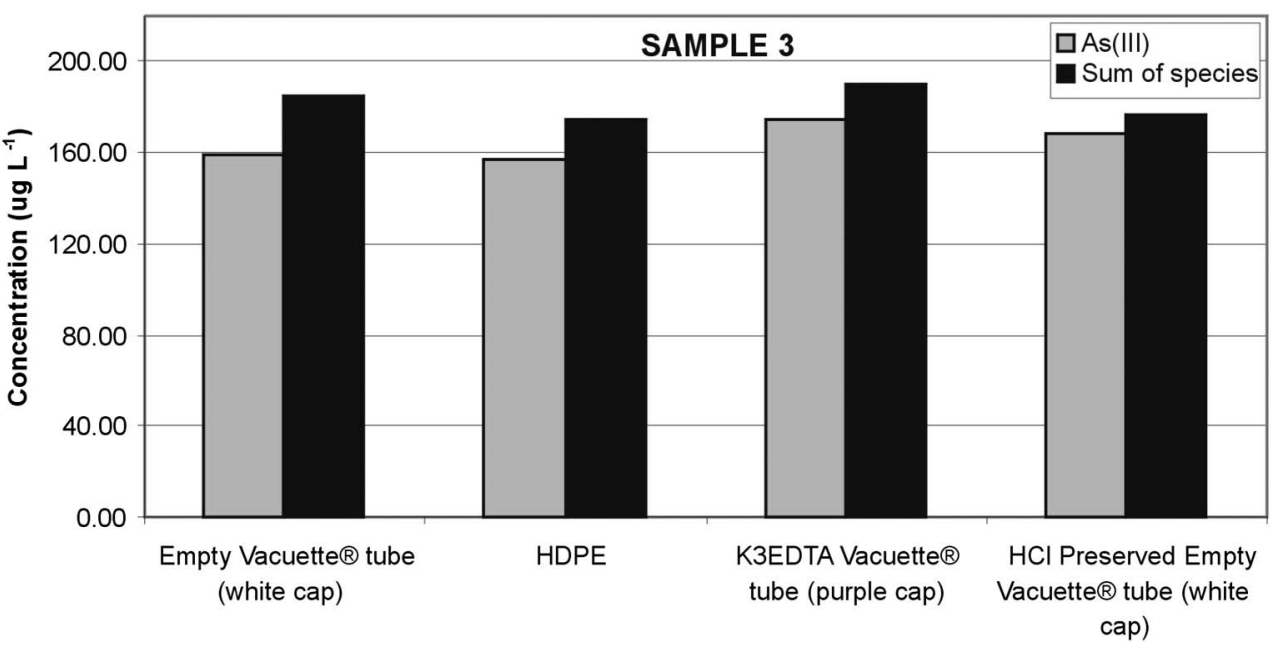

Sampling Media

Fig. 2. Arsenic species in unspiked environmental samples

amounts of arsenic in the three samples. Similarly, the total aluminum (Al) levels ranged between 2.27 and $28.73 \mu \mathrm{g} \mathrm{L}^{-1}$ for Sample 1; between 1.05 and $16.40 \mu \mathrm{g} \mathrm{L}^{-1}$ for Sample 2; and between 0.99 and $14.57 \mu \mathrm{g} \mathrm{L}^{-1}$ for Sample 3. The total Fe levels ranged between 12.36 and $213.24 \mu \mathrm{g} \mathrm{L}^{-1}$ for Sample 1; between
49.91 and $524.78 \mu \mathrm{g} \mathrm{L}^{-1}$ for Sample 2; and between 1,624.12 and $2,860.67 \mu \mathrm{g} \mathrm{L}^{-1}$ for Sample 3. These results revealed that Sample 3 contains high amounts of total iron, which were important for the presence and relative distribution of arsenic species. Finally, the total $\mathrm{Mn}$ amounts ranged between 1,117.21 and 1,275.19 $\mu \mathrm{g} \mathrm{L}^{-1}$ 
for Sample 1; between 1,491.39 and 1,679.59 $\mu \mathrm{g} \mathrm{L}^{-1}$ for Sample 2; and between $1,404.60$ and $1,624.33 \mu \mathrm{g} \mathrm{L}^{-1}$ for Sample 3 .

\section{Unspiked and Spiked Samples}

The results of arsenic speciation in the unspiked environmental samples are provided in Table 3. These samples have an average sum of species of $39.44,108.51$, and $181.65 \mu \mathrm{g} \mathrm{L}^{-1}$ arsenic levels, which correspond well with the total arsenic levels in these samples (37.17, 100.39, and $172.06 \mu \mathrm{g} \mathrm{L}^{-1}$, respectively). These values verify that the IC-ICP-MS method recovered all As species in the samples. The dominant arsenic species in these samples also demonstrated differences, as indicated by the oxygen redox potential (ORP) values given in Table 2. Consequently, Samples 2 and 3 have As(III) dominance, with average levels of 89.09 and $90.61 \%$ in all sample containers, respectively (Fig. 2). On the other hand, As(V) was dominant in Sample 1 (with an average $>97 \%$ in all sample containers) because this groundwater sample was withdrawn from an aquifer with a high ORP level (Fig. 2). A comparison of the sample containers shows that the HDPE bottle with no preservative typically demonstrated the lowest values in all samples, except Sample 2 (Fig. 2).

All environmental samples were spiked with corresponding As (III) levels (Table 3). The results of arsenic speciation and the corresponding recovery values are presented in Table 3. Here, As(III) and $\mathrm{As}(\mathrm{V})$ were the only species found in the samples and no organic species were detected. The sum of the two inorganic species is presented as the sum of species in Table 3, which provided comparable results for total arsenic measurements with relative percent differences (RPD) less than $20 \%$ for all samples (not shown), calculated according to

$$
\mathrm{RPD}=\frac{\mid \text { Sum of species }- \text { Total } \mid}{\frac{(\text { Sum of species }+ \text { Total })}{2}} \times 100
$$

where Sum of species $=$ sum of As(III) and As(V) detected in the sample; and Total $=$ total arsenic level measured separately in the same sample. Another measure for testing the effectiveness of the preservation method is to evaluate the percent recovery values for arsenite:

$$
\operatorname{Recovery}(\%)=\frac{C_{\text {sample }}-C_{\text {blank }}}{C_{\text {spike }}} \times 100
$$

where $C_{\text {sample }}=$ concentration of arsenite detected in the spiked sample (i.e., all even-numbered trials in Table 3 ); $C_{\text {blank }}=$ concentration of arsenite detected in the unspiked sample (i.e., all odd-numbered trials in Table 3); and $C_{\text {spike }}=$ corresponding concentration of $\mathrm{As}(\mathrm{III})$ added to the sample. Following this formula, higher percent recovery values correspond to better recovery of the field-spiked arsenic species under laboratory conditions.

According to the results given in Table 3, the percent recoveries of the field-spiked As(III) under laboratory conditions demonstrated that the vacuum tubes can stabilize the spiked As(III) in the samples. The percent recovery of As(III) has shown to vary between 92.9 and $103.8 \%$ for empty Vacuette tubes; between 32.2 and $107.2 \%$ for HDPE; between 96.6 and $111.1 \%$ for K3EDTA Vacuette tubes; and between 91.9 and $104.4 \%$ for $\mathrm{HCl}$ preserved, empty Vacuette tubes. With the exception of the HDPE bottles that contained no preserving agent, these results are extremely promising for arsenic speciation analysis. The common characteristic of all Vacuette tubes was that they all remained under vacuum conditions, which was probably the primary reason for preventing the oxidation of $\mathrm{As}(\mathrm{III})$ to $\mathrm{As}(\mathrm{V})$. Thus, the exposure to 


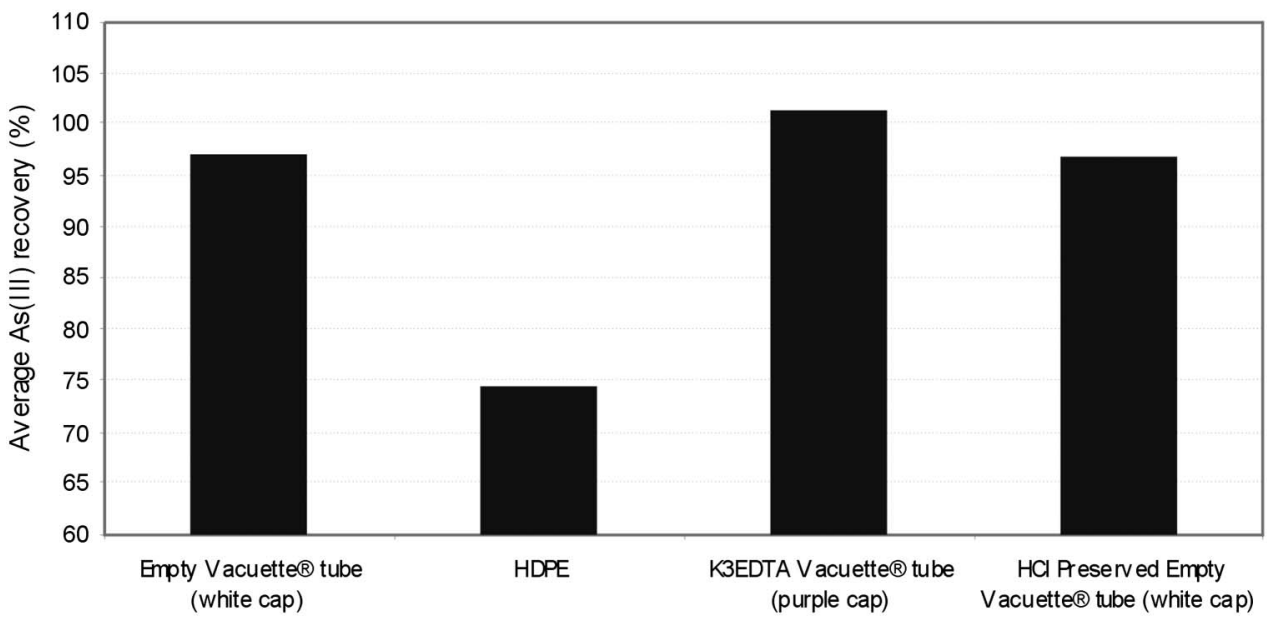

Sampling Media

Fig. 3. Average $\mathrm{As}(\mathrm{III})$ recovery rates with respect to sampling media

oxidizing agents was verified to be one of the most important parameters that results in deteriorating the integrity of the sample.

The statistical summary of the results is presented in Table 4. As shown in Fig. 3, Vacuette tubes provided very good recovery rates, all of which exceeded $97 \%$. The HDPE media, on the other hand, had an average As(III) recovery rate of $74.9 \%$ and was shown to be the least efficient of all media used in this study. A direct comparison between the empty Vacuette tubes and HDPE media showed that sampling into a container that is originally under vacuum improves the recovery ratios. Thus, if no chemical preservative is added to a sample, the presence of oxygen may cause As(III) oxidation to $\mathrm{As}(\mathrm{V})$.

A comparison of all three Vacuette tubes demonstrated very close recovery rates, which may be considered to be within the analytical error. However, the K3EDTA Vacuette tube performed slightly better in absolute terms and was closer to a $100 \%$ recovery value than the empty Vacuette tube and the empty $\mathrm{HCl}$ preserved Vacuette tube. In addition, the $\mathrm{As}(\mathrm{V})$ concentrations for the K3EDTA tubes were lower in the spiked samples than in the other containers, suggesting that the oxidation was lower in the K3EDTA tubes. However, this finding may also be related to the analysis techniques implemented in the laboratory. Hence, regardless of the preserving agent used, good recovery rates were obtained. Even in the empty Vacuette tube with no preserving agent, average recovery ratios of approximately $97 \%$ were achieved, strengthening the presumption that sample collection into vacuum tubes can preserve arsenic speciation in samples.

\section{Conclusions}

Arsenic speciation analysis is an increasingly popular area of research, primarily because of the differences in the toxicity levels of the species and their associated health impacts. The determination of total arsenic levels in water resources is no longer sufficient to accurately characterize the risk levels associated with human exposure to these waters. Consequently, accurate quantification of arsenic species present in samples is becoming a priority in many water quality monitoring studies. From a geochemical point of view, preserving the original species distribution in the sample until analysis is still considered to be a major difficulty because of the rapid oxidation potential of inorganic species [particularly As(III)] and the possibility of the integration of organic species into the biochemical reactions, which may alter the original distribution of species.

The methods implemented in this study are used to test the applicability of different sample collection/storage containers on the accuracy of speciation results under different water quality conditions. The effect of pressure conditions and the preserving agent in the sampling media were assessed with a laboratory speciation method. The amount of field-spiked As(III) recovered in the laboratory showed the overall performance of the sampling containers and the preserving agent. It was found that preserving the sample under vacuum conditions significantly improved the preservation of original species distribution in the water matrix, relative to sampling without preservatives. Thus, higher laboratory recovery rates were achieved with field-spiked samples in vacuum tubes. Obviously, the amount of time between sampling and analysis is a key parameter in obtaining the best results, and it is known that this time period should be kept as minimum as possible. However, when this condition is not met for some reason, storing the samples in vacuum conditions under airtight, cool, and dark settings is shown to be crucial for achieving more accurate results than if an unpreserved sampling is used. In addition, using a preserving agent such as EDTA or $\mathrm{HCl}$ is a well-known technique to preserve the sample. In this study, the differences between recovery ratios for these preserving agents did not provide major differences. EDTA performed slightly better, which may be attributed to the analysis technique. Nevertheless, using a commonly accepted preserving agent always has added benefits for samples representing more extreme conditions.

The current conclusions are based on groundwater samples that had total As levels in the order of 100-200 $\mu \mathrm{g} \mathrm{L}^{-1}$. Therefore, these conclusions are relevant for total As concentration ranges used in this study. In this study, no distinct relationship between concentration (Samples 1, 2, and 3) and sample preservation method (Sample Codes A, B, C, and D) is apparent for this order of magnitude. For lower or higher As levels, the relationship between recovery percentages and sampling preservation methods may be different. Therefore, it may be worthwhile for future studies to compare field preservation methods for other As concentration levels.

Overall, the use of a special sample collection media that is similar, in principle, to blood collection tubes provides promising results. Sample collection is just as simple as collecting samples into HDPE bottles; if K3EDTA tubes are to be used, the preservative reagents are already in the tubes. Because the total amount of liquid 
is limited to $10 \mathrm{~mL}$ or less, the use of such tubes has the added advantage of reduced sample volumes, which is typically a concern for large scale monitoring programs. Thus, shipping these sample tubes to the laboratory should be significantly cheaper than shipping HDPE bottles. Based on the findings reported in this study, these sterile vacuum tubes can better fit the requirements of water quality sampling than collection and storage without employing preservation methods. The use of such media is shown to provide promising results with regard to the speciation of arsenic. In that regard, the proposed technique should perform satisfactorily when oxygen is the major cause of the deterioration of sample integrity. Therefore, this finding may also be extended to preserve the speciation of other elements such as selenium, chromium, or antimony, or in measuring parameters such as nitrite or nitrate, which are subject to deterioration as a result of aerobic biochemical processes. Nevertheless, this hypothesis needs to be verified by future research under a wide spectrum of sample types and water quality conditions.

\section{Acknowledgments}

This study is funded by the Scientific and Technological Research Council of Turkey (TÜBİTAK) through project number 109Y029.

\section{References}

Abernathy, C. O., Thomas, D. J., and Calderon, R. L. (2003). "Health effects and risk assessment of arsenic." J. Nutr., 133(5), 1536S-1538S.

Agency for Toxic Substances, and Disease Registry (ATSDR). (2007). Toxicological profile for arsenic, U.S. Dept. of Health and Human Services, Public Health Service, Atlanta.

Akter, K. F., Owens, G., Davey, D. E., and Naidu, R. (2005). "Arsenic speciation and toxicity in biological systems." Rev. Environ. Contam. Toxicol., 184, 97-149.

Bednar, A. J., Garbarino, J. R., Ranville, J. F., and Wildeman, T. R. (2002). "Preserving the distribution of inorganic arsenic species in groundwater and acid mine drainage samples." Environ. Sci. Technol., 36(10), 2213-2218.

Bhattacharya, P., Welch, A. H., Stollenwerk, K. G., McLaughlin, M. J., Bundschuh, J., and Panaullah, G. (2007). "Arsenic in the environment: Biology and chemistry.” Sci. Total Environ., 379(2-3), 109-120.

Bissen, M., and Frimmel, F. H. (2003). "Arsenic-A review. Part I: Occurrence, toxicity, speciation, mobility." Acta Hydrochim. Hydrobiol., 31(1), 9-18.

Cullen, W. R., and Reimer, K. J. (1989). "Arsenic speciation in the environment." Chem. Rev., 89(4), 713-764.

EPA. (1999). Guidelines for exposure assessment, Risk Assessment Forum, Washington, DC.

Gallagher, P. A., Schwegel, C. A., Wei, X., and Creed, J. T. (2001). "Speciation and preservation of inorganic arsenic in drinking water sources using EDTA with IC separation and ICP-MS detection.” J. Environ. Monit., 3(4), 371-376.

Garbarino, J. R., Bednar, A. J., and Burkhardt, M. R. (2002). Methods of analysis by the U.S. Geological Survey National Water Quality
Laboratory arsenic speciation in natural-water samples using laboratory and field methods, USGS, Denver.

Gong, Z., Lu, X., Ma, M., Watt, C., and Le, X. C. (2002). "Arsenic speciation analysis." Talanta, 58(1), 77-96.

Grenier Bio-One. (2007). "Preanalytics." 〈http://www.gbo.com/en/ index_1585.php〉 (Aug. 5, 2012).

Gunduz, O., Simsek, C., and Hasozbek, A. (2010). "Arsenic pollution in the groundwater of Simav Plain, Turkey: Its impact on water quality and human health." Water Air Soil Pollut., 205(1), 43-62.

Hung, D. Q., Nekrassova, O., and Compton, R. G. (2004). "Analytical methods for inorganic arsenic in water: A review." Talanta, 64(2), 269-277.

Jain, C. K., and Ali, I. (2000). "Arsenic: Occurrence, toxicity and speciation techniques." Water Res., 34(17), 4304-4312.

Kapaj, S., Peterson, H., Liber, K., and Bhattacharya, P. (2006). "Human health effects from chronic arsenic poisoning: A review." J. Environ. Sci. Health Part A, 41(10), 2399-2428.

Kim, Y.-T., Yoon, H., Yoon, C., and Woo, N.-C. (2007). "An assessment of sampling, preservation, and analytical procedures for arsenic speciation in potentially contaminated waters." Environ. Geochem. Health, 29(4), 337-346.

Kumar, A. R., and Riyazuddin, P. (2010). "Preservation of inorganic arsenic species in environmental water samples for reliable speciation analysis." Trends Anal. Chem., 29(10), 1212-1223.

Kumaresan, M., and Riyazuddin, P. (2001). "Overview of speciation chemistry of arsenic." Curr. Sci., 80(7), 837-846.

Le, X. C., Yalcin, S., and Ma, M. (2000). "Speciation of submicrogram per liter levels of arsenic in water: On-site species separation integrated with sample collection." Environ. Sci. Technol., 34(11), 2342-2347.

Markley, C., and Herbert, B. (2009). "Arsenic risk assessment: The importance of speciation in different hydrologic systems." Water Air Soil Pollut., 204(1), 385-398.

Melamed, D. (2005). "Monitoring arsenic in the environment: A review of science and technologies with the potential for field measurements." Anal. Chim. Acta, 532(1), 1-13.

Miller, G. P., Norman, D. I., and Frisch, P. L. (2000). "A comment on arsenic species separation using ion exchange." Water Res., 34(4), $1397-1400$

Oliveira, V., Sarmiento, A. M., Gomez-Ariza, J. L., Nieto, J. M., and Sanchez-Rodas, D. (2006). "New preservation method for inorganic arsenic speciation in acid mine drainage samples." Talanta, 69(5), $1182-1189$.

Samanta, G., and Clifford, D. A. (2006). "Preservation and field speciation of inorganic arsenic species in groundwater." Water Qual. Res. J. Can., 41(2), 107-116.

Smedley, P. L., and Kinniburgh, D. G. (2002). "A review of the source, behaviour and distribution of arsenic in natural waters." Appl. Geochem., 17(5), 517-568.

Smith, A. H., Lingas, E. O., and Rhman, R. (2000). "Contamination of drinking-water by arsenic in Bangladesh: A public health emergency." Bull. World Health Organ., 78(9), 1093-1103.

Tapio, S., and Grosche, B. (2006). "Arsenic in the aetiology of cancer." Mutat. Res. Rev. Mutat. Res., 612(3), 215-246.

Terlecka, E. (2005). "Arsenic speciation analysis in water samples: A review of the hyphenated techniques." Environ. Monit. Assess., 107(1), 259-284.

Yalcin, S., and Le, X. C. (2001). "Speciation of arsenic using solid phase extraction cartridges." J. Environ. Monit., 3(1), 81-85. 